\title{
Persistent Current in a Ring Coupled to an External Fermionic Reservoir
}

\author{
Bernhard Wunsch and Alexander Chudnovskiy* \\ I. Institute of Theoretical Physics, University of Hamburg, Jungiusstr. 9, D-20355 Hamburg, Germany
}

\begin{abstract}
We study the energy spectrum and the persistent current in an ideal one-dimensional mesoscopic ring coupled to a fermionic reservoir. We find that the tunnel coupling in general leads to the suppression of the persistent current. However, with increasing coupling, the effective level structure of the ring coupled to the reservoir changes and quasistates with a sharp eigenenergy develop. Depending on the number of ring states coupled to the reservoir this results in a nonzero persistent current even at very large tunneling between the ring and the reservoir.
\end{abstract}

PACS numbers: 73.21.Ra, 73.23.-b

Experiments on mesoscopic rings enable to study quantum effects based on phase coherence like the AharonovBohm effect ${ }^{1,2}$ and persistent currents 3.4 . As phase coherence is the precondition of these phenomena, the influence of decoherence is of major interest. Recently, the suppression of quantum coherence in a mesoscopic system due to its coupling to an external macroscopic reservoir attracted much attention ${ }^{5}$. If a small mesoscopic system (quantum dot, quantum ring) is coupled by tunneling to an external reservoir of fermions (a lead) a phenomenon of level attraction is known to occur, which results in changes of occupation numbers, statistics of energy levels, and eventually the transport properties through the mesoscopic device. In the present paper we investigate the effects of the level attraction due to the tunnel coupling to an external reservoir on the persistent current in a mesoscopic ring.

A ring coupled to a reservoir was investigated previously within the scattering matrix approach ${ }^{6}$, in which the ring is coupled via an ideal wire to the dissipative reservoir. However, the development of long living states for strong coupling was not discussed there.

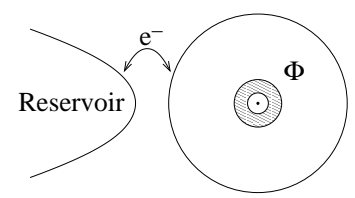

FIG. 1: Setup for a coupled ring described by the tunnel Hamiltonian formalism

The setup studied in this work is shown in figure 1 Since the main purpose of this paper is to study the influence of decoherence introduced by a tunnel contact to a fermionic reservoir (a lead) we confined ourselves to an independent spinless electron model and assumed the ring to be one-dimensional. This simple model captures already the main features of the energy spectrum and the persistent current measured on rings in the ballistic transport regime ${ }^{3}$. The Hamiltonian of our model has the following form:

$$
\begin{aligned}
\hat{H}= & \sum_{m} \varepsilon_{m} \hat{a}_{m}^{+} \hat{a}_{m}+\sum_{r} E_{r} \hat{b}_{r}^{+} \hat{b}_{r}+ \\
& +\sum_{m, r}\left(t_{m, r} \hat{a}_{m}^{+} \hat{b}_{r}+\text { h.c. }\right)
\end{aligned}
$$

where $\hat{a}_{m}^{+}$and $\hat{a}_{m}\left(\hat{b}_{r}^{+}\right.$and $\left.\hat{b}_{r}\right)$ are the creation and annihilation operators for electrons in the ring (reservoir) with quantum number $m(r)$. The eigenfunctions of the isolated ring are given by $\phi_{m}=e^{i m \varphi}$, where $m$ denotes the angular momentum in the ring. The corresponding eigenenergies are given by $\varepsilon_{m}=4 E_{0}\left(m+\frac{\Phi}{\Phi_{0}}\right)^{2}$, where $\Phi$ denotes the magnetic flux through the ring, $\Phi_{0}=\frac{h}{e}$ represents the magnetic flux quantum. The energy scale is given by $E_{0}=\frac{\hbar^{2}}{8 m^{*} R^{2}}$. The flux dependence of the Hamiltonian as well as the length of the ring is exclusively contained in the eigenenergies of the ring. The energies in the reservoir are denoted by $E_{r}$.

Following earlier discussion 8 it can be shown that for a small size of the contact between ring and reservoir, the tunneling matrix elements depend very weakly on the reservoir quantum number $r$. The dependence on the quantum number in the ring $m$ can be estimated as $t_{m, r} \propto \frac{2}{m} \sin m \varphi_{0}$, where $\varphi_{0}$ describes the angular size of the tunnel contact. In this paper we set the tunnel matrix elements constant for a given range of angular momenta of the ring eigenstates. The tunnel matrix elements for other ring states are set to zero.

We point out, that different ring states couple to the same states in the reservoir. This effective interaction between ring states will determine the behavior for strong coupling, as shown in the following.

Within the described model, a Dyson equation for the Green's function can be solved exactly for arbitrary tunneling strength. The retarded Green's function determines the spectral density of the ring states $S_{m}(E)=-\frac{1}{\pi} \operatorname{Im}\left(\mathcal{G}_{m}^{r e t}(E)\right)$, from which the total density of states (DOS) inside the ring $\rho(E)$ is obtained:

$$
\rho(E)=\sum_{m} S_{m}(E)=\frac{-\kappa \frac{\partial \xi(E)}{\partial E}}{\pi\left(1+\kappa^{2} \xi(E)^{2}\right)},
$$


with $\xi(E)=\sum_{m_{1}} \frac{1}{E-\varepsilon_{m_{1}}}$ and $\kappa=\pi \nu|t|^{2}$. The obtained results are also valid for the strong coupling regime, in which the energy scale given by the tunneling is of the order of or larger than the interlevel spacing between consecutive ring states. To avoid superimposing effects on the DOS in the ring due to the band structure of the reservoir we choose a constant density of states in the reservoir $\nu(E)=\nu$.

In the simplest case of only one ring state that couples to the reservoir, the DOS is given by a Lorentzian centered around the eigenenergy $\varepsilon_{0}$ of the isolated state $\rho(E)=\frac{\kappa}{\pi\left(\left(E-\varepsilon_{0}\right)^{2}+\kappa^{2}\right)}$ with the width given by the coupling energy $\kappa$.

The effect of coupling to the reservoir on the persistent current in the ring is investigated by calculating the current density. The latter is obtained by summing over the contributions of all ring states. As the current carried by an occupied isolated ring state is $I_{m}=-\frac{\partial \varepsilon_{m}}{\partial \Phi}$, the current density has the form

$$
j(E)=\sum_{m}-\frac{\partial \varepsilon_{m}}{\partial \Phi} S_{m}(E)=-\frac{\kappa \frac{\partial \xi(E)}{\partial \Phi}}{\pi\left(1+\kappa^{2} \xi(E)^{2}\right)} .
$$

The total current is then given by $I=\int_{-\infty}^{\infty} f(E) j(E) d E$, where $f(E)$ denotes the Fermi distribution.

Let us first assume, that the coupling between ring and reservoir is restricted to the two energetically lowest ring states. This can be motivated by selective tunneling with respect to the angular momentum of the ring states as discussed above. Furthermore for a magnetic flux close to $\Phi=\frac{\Phi_{0}}{2}$ this assumption is also a good approximation as long as the energy gap to the higher lying ring states is larger than the coupling energy $\kappa$. However we will not limit the coupling strength in the following discussion.

If only two ring states couple to the reservoir, the system can be well described by introducing two quasistates. For weak coupling, their DOS is given by:

$$
S_{1 / 2}(E)=\frac{\kappa}{\pi\left(\left(E_{a v} \pm \sqrt{\frac{(\Delta \varepsilon)^{2}}{4}-\kappa^{2}}\right)^{2}+\kappa^{2}\right)}
$$

with $\Delta \varepsilon=\varepsilon_{m_{1}}-\varepsilon_{m_{2}} ; \quad E_{a v}=E-\frac{\varepsilon_{m_{1}}+\varepsilon_{m_{2}}}{2}$. The strength of the coupling is characterized by the tunneling energy $\kappa$ defined above, which has to be compared to the interlevel spacing $\Delta \varepsilon$ of the coupled ring states. Without coupling, the quasistates coincide with the eigenstates of the isolated ring. The solid line in figure 2 shows the DOS in the weak coupling regime defined by $\frac{(\Delta \varepsilon)^{2}}{4}-\kappa^{2}>0$. In this regime the spectral densities of the quasistates broaden with increasing coupling, thereby approaching each other.

At the critical coupling $\kappa_{c}=\left|\frac{\Delta \varepsilon}{2}\right|$ the spectral densities of the quasistates are equivalent. The dashed line in figure 2 illustrates the DOS, if the coupling is increased into the strong coupling regime with $\kappa>\kappa_{c}$. One quasistate with a sharp eigenenergy develops and is represented by the sharp peak in the DOS with a width smaller than

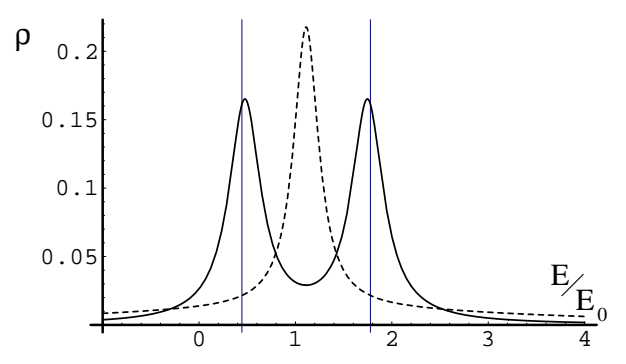

FIG. 2: DOS for two coupled states and fixed magnetic flux $\Phi=\frac{\Phi_{0}}{3}$ for weak coupling (full line, $\kappa=0.2 E_{0}$ ) and strong coupling (dashed line, $\kappa=1.5 E_{0}$ ). The grid lines show the eigenergies in the isolated ring.

$\kappa$. The other quasistate contributes to the DOS within a broad energy range of a width larger than $\kappa$. The described phenomenon can be related to the Dicke effect in optics ${ }^{7}$. The manifestation of the latter has been investigated recently in context of resonant scattering and resonant tunneling in solid state systems ${ }^{\underline{*}}$. The behavior of the DOS is well described by the spectral densities of the quasistates in the strong coupling regime:

$$
S_{1 / 2}(E)=\frac{\kappa \mp \sqrt{\kappa^{2}-\frac{(\Delta \varepsilon)^{2}}{4}}}{\pi\left(E_{a v}^{2}+\left(\kappa \mp \sqrt{\kappa^{2}-\frac{(\Delta \varepsilon)^{2}}{4}}\right)^{2}\right)} .
$$

It is important to notice that the energies of the isolated ring states depend on the magnetic flux, while the coupling to the reservoir is assumed to be independent of the magnetic flux. In particular, the eigenenergies are degenerate at $\Phi=n \frac{\Phi_{0}}{2}$, so that by changing the magnetic flux close to this degenerate value one finally enters the regime of strong coupling, for any nonzero coupling strength.

The energy of the long living state depends on the magnetic flux, as it is given by the average energy of the two coupled states. Therefore, the system shows AharonovBohm type behavior even in the strong coupling regime. Correspondingly the persistent current saturates in the limit of strong coupling and does not vanish as illustrated in figure 3 In the limit of strong coupling the long living state carries the current $I=\frac{I_{m_{1}}+I_{m_{2}}}{2}$, while the broad state carries a current of $I=\frac{I_{m_{1}}^{2}+I_{m_{2}}}{4}$. Therfore the value of the saturated persistent current is either $\frac{1}{4}$ or $\frac{3}{4}$ of the current in the isolated ring, depending on whether the Fermi energy lies below or above the energy of the long-living state.

A generalization of the simplified two level model is obtained by considering the coupling of more ring states to the reservoir. Thereby at least all states with an eigenenergy below the Fermi energy are coupled to the reservoir. In the strong coupling regime the system develops long living states between nearest neighbors whenever the tunneling energy $\kappa$ exceeds the interlevel spacing between the corresponding eigenenergies of the isolated ring. Like in 


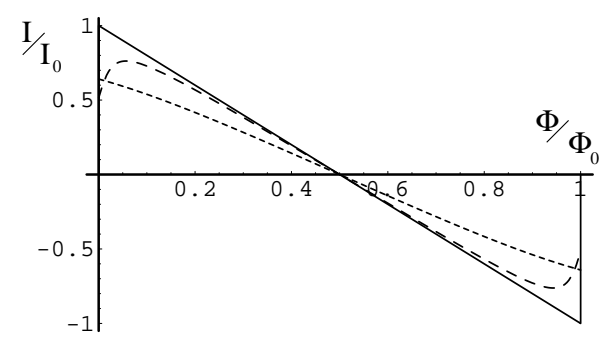

FIG. 3: Dependence of the persistent current on the magnetic flux for two coupled ring states. Parameters: $\mu=4 E_{0}$; full line: $\kappa=0$; long dashed line: $\kappa=0.2 E_{0}$; short dashed line: $\kappa=5 E_{0}$.

the case of two coupled levels, the persistent current saturates in the limit of strong coupling at a generally nonzero value. Thereby the saturation value of the persistent current depends strongly on the number of coupled states. It decreases with increasing number of coupled ring states, but it also shows an odd-even effect with the number of coupled states. Both features have their origin in the alternating sign of the current carried by consecutive ring states.

Now we consider the coupling of all ring states to the reservoir. According to our previous discussion about the tunnel matrix element this situation is realized in the limit of a point contact. It is an appealing feature of our model that an easy analytical formula for the DOS in the ring and the current density can be given for this limiting case:

$$
\rho(E)=\frac{\kappa}{\pi} \frac{\frac{1}{x} \sin x(\cos \Phi-\cos x)+1-\cos x \cos \Phi}{\kappa^{2}(\sin x)^{2}+\frac{4 E_{0}^{2} x^{2}}{\pi^{4}}(\cos \Phi-\cos x)^{2}}
$$

with $x=\pi \sqrt{\frac{E}{E_{0}}}$, thereby we have set $\hbar=e=c=1$. To obtain the DOS given in equation (6) we have used $\xi(E)=\sum_{m=-\infty}^{\infty} \frac{1}{E-\varepsilon_{m}}=\frac{\pi^{2}}{2 E_{0} x} \frac{\sin x}{\cos \Phi-\cos x}$.

For small coupling the DOS shows Lorentz broadened maxima around the eigenenergies of the isolated ring states with a width given by the coupling energy $\kappa$, in agreement with the coupling of two states. Figure 4 illustrates the development of the DOS with increasing coupling. The maxima in the DOS belonging to different ring states move together with increasing coupling and at a critical coupling new maxima are formed at $E=(2 m+1)^{2} E_{0}$. Thereby the critical coupling grows with energy proportional to $\sqrt{E}$, like the interlevel spacing between the eigenenergies of the isolated ring. At even stronger coupling additional quasistates are developed at $E=(2 m)^{2} E_{0}$. The peaks in the DOS corresponding to these quasistates are more and more pronounced with increasing coupling. Their energies have also a special meaning in the energy spectra of the isolated ring. $E=(2 n+1)^{2} E_{0}$ are the two fold degenerate eigenenergies for $\Phi= \pm \frac{\Phi_{0}}{2}$, while $E=(2 n)^{2} E_{0}$ are the

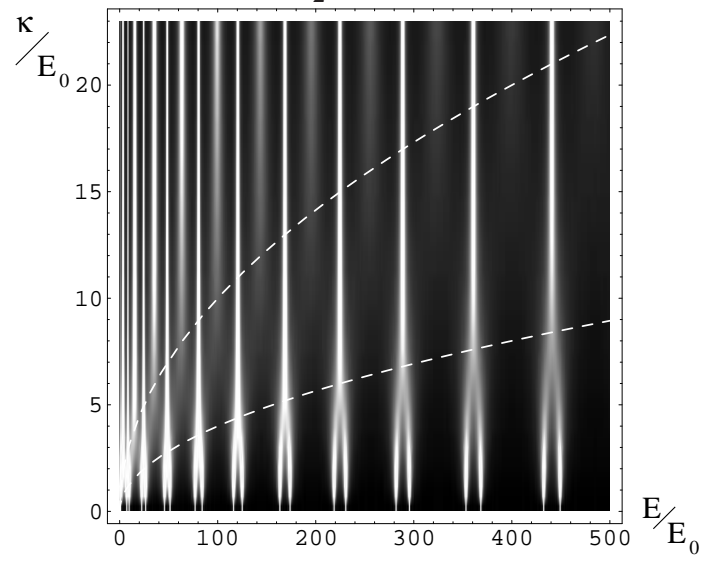

FIG. 4: Density plot of DOS as a function of the coupling for fixed magnetic flux $\Phi=0.4 \Phi_{0}$ (the magnitude of the DOS increases going from black to white). For weak coupling the maxima are located at the eigenenergies of the isolated ring states whereas for strong coupling quasistates at $E=n^{2} E_{0}$ develop. The critical coupling thereby depends on the energy as $\sqrt{E}$ with different prefactors for even or odd $\mathrm{n}$ as indicated by the dashed lines $(0.4 \sqrt{E}, \sqrt{E})$.

two fold degenerate eigenenergies for $\Phi=n \Phi_{0}$. For other values of the magnetic flux the energy spectrum is nondegenerate.

In contrast to the coupling of a finite number of ring states, the eigenenergies of the long living states in the strong coupling regime are now independent of the magnetic field, which evidences for the localization of those states in the ring. Consequently, the Aharonov-Bohm effect disappears, which is accompanied by a continuous suppression of the persistent current with increasing coupling as illustrated in figure 5

The authors are grateful to D. Pfannkuche for illuminating discussions. Financial support form SFB 508 is gratefully acknowledged.
* Electronic address: achudnov@physnet.uni-hamburg.de

${ }^{1}$ Y. Aharonov and D. Bohm, Phys. Rev.115, 485 (1959).

2 A. W. Holleitner, et al., Phys. Rev. Lett. 87, 256802 (2001); A. Fuhrer, et al., Nature 413, 822 (2001).

3 A. Lorke, et al., Phys. Rev. Lett. 84, 2223 (2000);D. Mailly et al., Phys. Rev. Lett. 70, 2020 (1993).

${ }^{4}$ E.M.Q. Jariwala, et al., Phys. Rev. Lett. 86, 1594 (2001).

5 A. L. Chudnovskiy and S. E. Ulloa, Phys. Rev. B 63,165316 (2001);A. L. Chudnovskiy and S. E. Ulloa, Physica E 12, 819 (2002). 


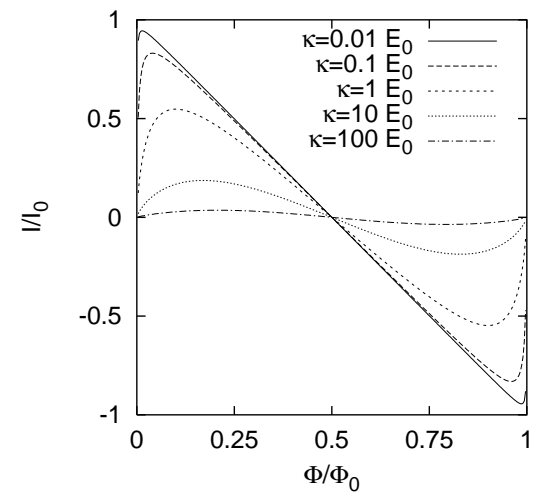

FIG. 5: Dependence of the persistent current on the magnetic flux for different coupling strengths and for coupling of all ring states. For all curves, there are five states below the Fermi energy $\mu=25 E_{0}$.
6 M. Büttiker Phys. Rev. B 32, 1846 (1985); Pier A. Mello, Phys. Rev. B 47, 16358 (1993).

7 R.H. Dicke, Phys. Rev. 89, 472 (1953); R.H. Dicke, Phys. Rev. 93, 99 (1954).

8 T.V. Shahbazyan and S.E. Ulloa, Phys. Rev. B 57, 6642 (1998). 